\title{
Torsades de Pointes Secondary to Excessive
}

\section{Loperamide Use}

\author{
Joseph S. Van Tuyl ${ }^{1}$, Gail Brock ${ }^{2}$, Kristen Bova Campbell ${ }^{2}$ and Sana M. Al-Khatib ${ }^{2}$ \\ 1. Department of Pharmacy Practice, St. Louis College of Pharmacy, 4588 Parkview Place, St. Louis, MO 63110 \\ 2. Department of Medicine, Duke University Hospital, DUMC 3850, Durham, NC 27710
}

\begin{abstract}
Loperamide is a common, over-the-counter, antidiarrheal medication that has been implicated in cases of ventricular tachycardia. A 32 year-old female patient with a history of opioid abuse and no prior history of cardiovascular disease experienced Torsades de pointes. An electrocardiogram at the time of hospital presentation revealed a prolonged QT interval at 636 msec. Diagnostic evaluation was unremarkable for any underlying cardiovascular pathology. Further investigation revealed the patient had been taking 30-40 loperamide $2 \mathrm{mg}$ tablets oral daily for 2 weeks prior to the onset of Torsades de pointes. At high doses, loperamide may prolong the QT interval through antagonism of the human ether-a-go-go potassium current, thus increasing the risk of ventricular arrhythmias. Clinicians should be cautious with administration of high doses of loperamide to all patients, especially patients at high risk for QT interval prolongation.
\end{abstract}

Key words: Torsades de pointes, loperamide, QT prolongation.

\section{Abbreviations}

$\begin{array}{ll}\text { ECG, } & \text { electrocardiogram; } \\ \text { HERG, } & \text { human ether-a-go-go; } \\ \text { ICD, } & \text { implantable cardioverter defibrillator; } \\ \text { LVEF, } & \text { left ventricular ejection fraction; } \\ \text { PVC, } & \text { premature ventricular contraction; } \\ \text { TdP, } & \text { Torsades de pointes; } \\ \text { VT, } & \text { ventricular tachycardia. }\end{array}$

\section{Introduction}

Loperamide is an over-the-counter anti-diarrheal medication and mu opioid receptor agonist. The chemical structure of loperamide is similar to fentanyl and methadone, mu opioid receptor agonists known to inhibit the cardiac HERG (human ether-a-go-go) potassium current [1]. The maximum recommended dose of loperamide is $16 \mathrm{mg}$ oral daily [2]. However, loperamide is frequently administered at doses far exceeding these recommendations and is reportedly used by patients with a history of illicit drug use to achieve euphoric effects [3].

Corresponding author: Joseph S. Van Tuyl, assistant professor. St. Louis College of Pharmacy 4588 Parkview Place St. Louis, MO 63110.
Case reports have associated high-dose loperamide administration with potentially fatal ventricular arrhythmias [4-7]. The loperamide package insert does not warn of a risk of QT interval prolongation or ventricular arrhythmias, and clinicians may be unaware of the potential complications of high-dose loperamide. This report describes the clinical course of a probable case of loperamide-induced TdP (Torsades de pointes) in a patient with a history of opioid addiction.

\section{Case}

A 32 year-old woman was transported to an outside hospital after she experienced cardiac arrest at home with return of spontaneous circulation after approximately 40 seconds. Upon presentation to the emergency department, an ECG (electrocardiogram) initially revealed ventricular bigeminy and a prolonged QT interval of $636 \mathrm{msec}$ (Table 1) without evidence of Wolff-Parkinson-White phenomenon or Brugada syndrome. Telemetry monitoring documented long-short intervals caused by PVCs (premature ventricular contractions), and R-on-T phenomenon led to polymorphic ventricular tachycardia on two separate 
occasions. Approximately six external defibrillations were required to restore sinus rhythm, and the patient received an amiodarone bolus $150 \mathrm{mg}$ and continuous infusion to suppress subsequent runs of non-sustained VT (ventricular tachycardia) (Fig. 1). After 24 hours, the patient continued to have recurrent episodes of non-sustained VT and was transitioned to a lidocaine continuous infusion at $1 \mathrm{mg} / \mathrm{min}$ on which she did not experience further ventricular arrhythmias.

A stress cardiac MRI revealed a mildly reduced LVEF (left ventricular ejection fraction) of $46 \%$ without evidence of ischemia or scarring on the myocardium. Cardiac enzymes were negative, and a left heart catheterization was unremarkable for coronary artery disease. The patient had mild hypokalemia and hypomagnesemia with serum potassium and magnesium levels of $3.4 \mathrm{mEq} / \mathrm{L}$ and 1.8 $\mathrm{mEq} / \mathrm{L}$ (Table 2), respectively. A urine toxicology screen was negative. The patient's past medical history was negative for cardiovascular disease and electrophysiologic abnormalities. Her third child was born approximately six months prior to this event without any documented post-partum cardiomyopathy.
Family history was unremarkable for sudden cardiac death, and the patient denied recent herbal supplement or stimulant use.

The patient was subsequently transferred to Duke University Hospital for further diagnostic evaluation and consideration of placement of an ICD (implantable cardioverter defibrillator). Her QTc at the time of transfer was $532 \mathrm{msec}$ (Table 1). Upon arrival to the hospital, the lidocaine infusion was continued at 1 $\mathrm{mg} / \mathrm{min}$ and metoprolol $25 \mathrm{mg}$ PO every 6 hours was initiated. An ECG revealed that the QTc interval continued to be prolonged at $517 \mathrm{msec}$. Telemetry monitoring was unremarkable for PVCs or runs of VT throughout her stay. Laboratory parameters remained within normal limits for the duration of the hospitalization. A repeat echocardiogram 2 days after the cardiac MRI revealed recovery of left ventricular function with a LVEF greater than 55\%. An ECG from an outside provider dated 2013 demonstrated a normal QTc interval of $429 \mathrm{msec}$, suggesting the presenting ventricular arrhythmia was secondary to a reversible etiology.

At this point in the admission, the patient revealed a
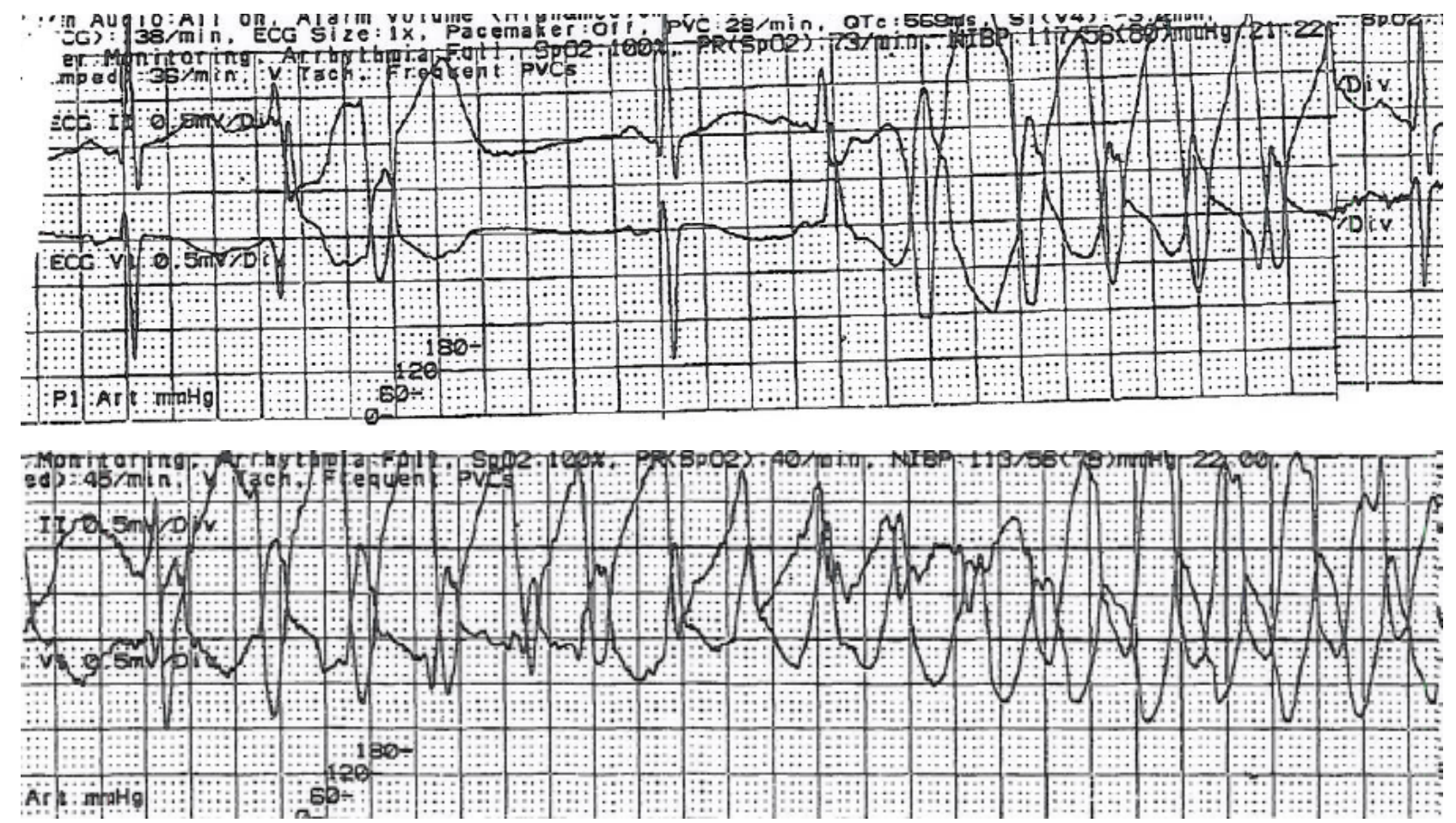

Fig. 1 Telemetry Documentation of TdP Onset. 
Table 1 12-Lead ECG parameters.

\begin{tabular}{llllllll}
\hline & \multicolumn{3}{c}{ Outside Hospital } & \multicolumn{5}{c}{ After Hospital Transfer } \\
\cline { 2 - 8 } & Day -4 & Day -3 & Day 0 & Day 1 & Day 3 & Day 37 & Day 44 \\
\hline Ventricular Rate, bpm $^{\dagger}$ & 90 & 88 & 65 & 67 & 66 & 76 & 83 \\
PR Interval, msec $^{\ddagger}$ & 170 & 176 & 150 & 144 & 128 & 138 & 152 \\
QRS Duration, msec $^{\ddagger}$ & 112 & 114 & 88 & 82 & 80 & 88 & 90 \\
QT Interval, msec $^{\ddagger}$ & 520 & 616 & 512 & 490 & 484 & 458 & 370 \\
QTc (Bezet), msec & 636 & 745 & 532 & 517 & 507 & $470^{\S}$ & 452 \\
\hline
\end{tabular}

†bpm = beats per minute; $\$ \mathrm{msec}=$ milliseconds.

§ECG completed while patient was receiving olanzapine.

Table 2 Serum potassium and magnesium concentrations.

\begin{tabular}{llllllll}
\hline & Day 1 & Day 2 & Day 3 & Day 4 & Day 5 & Day 6 & Day 7 \\
\hline Serum Potassium, $\mathrm{mEq} / \mathrm{L}$ & 3.4 & 4.3 & - & 3.6 & 3.8 & 4.0 & 4.1 \\
Serum Magnesium, $\mathrm{mEq} / \mathrm{L}$ & 1.8 & 2.4 & - & 2.0 & 2.1 & 2.3 & 1.9 \\
\hline
\end{tabular}

history of opioid addiction which had previously been controlled with buprenorphine/naloxone. Approximately 6 weeks before the cardiac arrest event, the patient abruptly discontinued buprenorphine/naloxone. She began experiencing symptoms of opioid withdrawal (e.g. diarrhea) and also reported lightheadedness and hot flashes. She started self-treatment for the withdrawal symptoms with loperamide. The patient reported taking 30-40 tablets of loperamide $2 \mathrm{mg}$ tablets daily for approximately 2 weeks prior to the index cardiac arrest event.

The lidocaine infusion and metoprolol were discontinued, and the patient remained free of arrhythmias for the duration of the hospitalization before being transferred to a drug rehabilitation facility. She was seen in clinic one month after discharge and found to have a prolonged QTc of $470 \mathrm{msec}$ (manual measurement) while receiving treatment with olanzapine. The olanzapine was discontinued and a follow-up ECG showed manually measured QT and QTc of 370 msec and 452 msec, respectively (Table 1).

The Naranjo probability scale is an adjudicated questionnaire that estimates the likelihood of an adverse drug reaction [8]. A score provided by the questionnaire estimates the probability of an adverse drug reaction as definite (score $\geq 9$ ), probable (score 5 to 8 ), possible (score 1 to 4 ), or doubtful (score $\leq 0$ ). According to the Naranjo scale, the likelihood of loperamide precipitating this ventricular arrhythmia is probable (score 7 out of 10 ).

\section{Discussion}

Several cases have been reported that implicate loperamide use as the cause of life-threatening ventricular arrhythmias [4-7]. A case series describes five patients who experienced electrophysiologic abnormalities after overuse of loperamide in which most of the patients were taking greater than $100 \mathrm{mg}$ of loperamide daily. None of the patients had underlying structural or functional cardiovascular conditions that would predispose to ventricular conduction disturbances. Three of the patients experienced polymorphic ventricular tachycardia. Of these cases, two had ventricular arrhythmias refractory to administration of amiodarone, lidocaine, magnesium, sodium bicarbonate, or fat emulsion. However, suppression of further ventricular arrhythmias was achieved through overdrive pacing with a transvenous pacemaker or pharmacologic pacing with an isoproterenol infusion titrated to a heart rate of 90 beats per minute. For each case, discontinuation of loperamide resulted in normalization of the QRS and QT intervals, and patients remained free of arrhythmias prior to hospital discharge [4].

Another report describes a 54-year-old woman hospitalized for syncope and found to have sinus 
pauses with intermittent episodes of VT. The patient had a history of type II diabetes mellitus and chronic diarrhea secondary to a cholecystectomy several years prior. Initial diagnostic testing was unremarkable for identifiable causes of ventricular ectopy. A medication history revealed the patient had been self-treating the diarrhea for several years with escalating doses of loperamide up to $144 \mathrm{mg}$ daily. Subsequent ECGs revealed sinus arrest, a slow junctional escape rhythm, and frequent premature ventricular contractions. The rhythm degenerated into polymorphic ventricular tachycardia requiring cardiopulmonary resuscitation. Intravenous amiodarone and lidocaine infusions were started, but the patient continued to have several runs of sustained VT. The ventricular ectopy was eventually suppressed by transvenous pacing at 90 beats per minute. The pacing requirement decreased over the course of 72 hours, and a follow-up ECG exhibited sinus rhythm, first-degree AV block, and QT interval prolongation at $500 \mathrm{msec}$. An ICD was implanted prior to discharge, and the patient remained free of arrhythmias at two months of follow-up [5].

Enakpene et al. described a unique case of a 25 -year-old woman with an unremarkable past medical history initially diagnosed with a long QT syndrome and implanted with a dual-chamber ICD after recurrent hospitalizations for gastrointestinal discomfort and syncope accompanied by QRS and QT interval prolongation. Six weeks after implantation of the ICD, the patient was hospitalized with bradycardia and hypotensive shock that devolved into polymorphic ventricular tachycardia. Further episodes of polymorphic ventricular tachycardia accompanied by a prolonged QRS duration led the physicians to suspect a sodium-channel blocker toxicity, and a bicarbonate infusion was initiated with minimal improvement in QRS duration. Additional investigation revealed several empty bottles of loperamide at the patient's residence, and toxicologic screening demonstrated a significantly elevated loperamide level of $32 \mathrm{ng} / \mathrm{mL}$. The patient continued to receive supportive care and intravenous infusions of bicarbonate and fat emulsion. The ventricular arrhythmias resolved within 1 week of discontinuation of loperamide. Unfortunately, the patient later died from cardiopulmonary arrest secondary to continued loperamide abuse [6].

Finally, a report by Marzec et al. illustrated the profound effect of loperamide on QT interval prolongation by reporting on a 26-year-old man admitted with TdP with a QTc interval greater than 700 msec and evidence of systolic dysfunction on an echocardiogram. The patient had been taking 100-250 $\mathrm{mg}$ of loperamide daily with concomitant cimetidine $400 \mathrm{mg}$ daily for several months prior to hospital presentation. After loperamide discontinuation, the patient remained free of ventricular arrhythmias, systolic function normalized, and a repeat ECG revealed a QTc interval of $420 \mathrm{msec}$ two months later [7].

The aforementioned cases possess remarkably similar characteristics to the current case report. The patients in each case were relatively healthy without documented evidence of cardiovascular disease that would predispose patients to the development of ventricular arrhythmias. The instances in which cardiac dysfunction were identified on echocardiography resolved after discontinuation of loperamide. All of the ventricular arrhythmias were accompanied by a markedly prolonged QT/QTc interval which subsequently normalized after discontinuation of the medication. Additionally, the ventricular arrhythmias that were likely induced by loperamide appeared to be refractory to pharmacologic interventions, and some required overdrive pacing to suppress ventricular ectopy while the electrophysiologic abnormalities resolved.

The over-the-counter anti-diarrheal medication loperamide is a piperadine derivative with agonist activity at mu opioid receptors that produces its pharmacologic effect through anti-secretory activity and decreasing gastrointestinal motility. The distribution of loperamide to the central nervous 
system is minimal and the associated pharmacologic effects such as respiratory depression and analgesia are absent under normal conditions. The medication is metabolized to its inactive form through $\mathrm{N}$-demethylation and several isozymes of cytochrome P450 including 3A4 and 2D6 [9]. Increased exposure to loperamide may occur after administration of potent cytochrome P450 3A4 and 2D6 inhibitors and inhibitors of P-glycoprotein predisposing patients to the central nervous system effects of opioids. P-glycoprotein inhibition has been observed to increase the distribution of loperamide into the central nervous system and may result in respiratory depression, analgesia, and euphoria at sufficient doses [10-12]. Loperamide is administered in $2 \mathrm{mg}$ increments as needed for control of diarrhea up to a maximum recommended dose of $16 \mathrm{mg}$ per day. Under usual dosages, the medication has a half-life of approximately 9-13 hours. Prolonged half-lives approaching 35-40 hours have been documented with doses greater than or equal to $16 \mathrm{mg}$ and may also occur with inhibition of the cytochrome P450 system and P-glycoprotein [13].

Evidence indicates that loperamide use may be increasing in patients with a history of opioid addiction to control withdrawal symptoms. Review of submissions to a website that allows discussion of illicit drug use noted an increase in comments about the use of loperamide for control of opioid withdrawal symptoms around 2010-2011. A minority of the submissions discussed the use of loperamide to achieve the theorized euphoric effects of the medication. More importantly, though, most of the posts indicated individuals were taking an average dose of $70 \mathrm{mg}$ per day, and a few instances described using doses ranging from 100-200 mg daily to control symptoms [3]. These doses far exceed the maximum recommended dose of loperamide and correlate with the doses associated with QT interval prolongation and ventricular arrhythmias in case reports.

The exact mechanism of loperamide-induced QT interval prolongation remains unknown, but potential mechanisms have been proposed on the basis of similarities between loperamide and other opioids. The opioid agonists methadone and fentanyl, among others, have demonstrated an ability to inhibit the HERG potassium current [1]. These opioid agonists have a similar chemical structure to loperamide, and thus, HERG potassium current antagonism may represent a plausible mechanism for QT interval prolongation. The prolonged repolarization phase, indicated by the QT interval, increases transmural dispersion of repolarization in the ventricular myocardium and increases the risk of early afterdepolarizations which precipitate TdP [14].

The risk of QT interval prolongation and TdP is not mentioned in the loperamide package insert. [2] The collective evidence, however, indicates that loperamide, when used at very high doses, causes QT interval prolongation and predisposes patients to the development of ventricular arrhythmias. These reports occurred in patients without any underlying conduction disturbances or cardiovascular disease. Loperamide package labeling should be amended to include a warning for the risk of ventricular arrhythmias when loperamide is administered at doses exceeding $16 \mathrm{mg}$ daily. Clinicians should exercise caution with the administration of high doses of loperamide to all individuals, especially those considered to be at high risk for QT interval prolongation. Patients at high risk include the elderly, female sex, patients with a history of electrolyte abnormalities (specifically hypokalemia or hypomagnesemia), and patients receiving concomitant medications that prolong the QT interval or inhibit loperamide metabolism [15]. Additionally, clinicians should be proactive in discussing loperamide use in patients with a history of opioid dependence and/or illicit drug use.

\section{Conclusion}

Several published cases report the onset of ventricular arrhythmias after administration of high 
doses of loperamide [4-7]. We describe a 32 year-old woman who experienced TdP after excessive use of loperamide to manage symptoms of opioid withdrawal. High-dose loperamide can prolong the QT interval and increase the risk of ventricular arrhythmias. Clinicians should be cautious with the administration of high-dose loperamide to all patients, particularly those predisposed to QT interval prolongation and with a history of illicit drug use.

\section{References}

[1] Katchman, A. N., McGroary, K. A., Kilborn, M. J., Kornick, C. A., Manfredi, P. L., Woosley, R. L., and Ebert, S. N. 2002. "Influence of Opioid Agonists on Cardiac Human Ether-A-Go-Go-Related Gene K+ Currents." JPET 303: 688-94.

[2] Imodium [package insert]. 2008. Raritan, NJ: Janssen Pharmaceutica Inc.

[3] Daniulaityte, R., Carlson, R., Falck, R., Cameron, D., Perera, S., Chen, L., and Sheth, A. 2013. "I Just Wanted to Tell You that Loperamide Will Work: A Web-Based Study of Extra-Medical Use of Loperamide." Drug Alcohol Depend 130: 241-4.

[4] Marraffa, J. M., Holland, M. G., Sullivan, R. W., Morgan, B. W., Oakes, J. A., Wiegand, T. J., and Hodgman, M. J. 2014. "Cardiac Conduction Disturbance after Loperamide Abuse." Clin Toxicol 52: 952-7.

[5] Spinner, H. L., Lonardo, N. W., Mulamalla, R., and Stehlik, J. 2015. "Ventricular Tachycardia Associated with High-Dose Chronic Loperamide Use." Pharmacotherapy 35: 234-8.

[6] Enakpene, E. O., Riaz, I. B., Shirazi, F. M., Raz, Y., and Indik, J. H. 2015. "The Long QT Teaser: Loperamide Abuse.” Am. J. Med. 05, 19.
[7] Marzec, L. N., Katz, D. F., Peterson, P. N., Thompson, L. E., Haigney, M. C., and Krantz, M. J. 2015. "Torsade de Pointes Associated with High-Dose Loperamide Ingestion." J. Innov. Cardiac. Rhythm. Manage. 6: 1897-9.

[8] Naranjo, C. A., Busto, U., Sellers, E. M., Sandor, P., Ruiz, I., Roberts, E. A., Janecek, E., Domecq, C., and Greenblatt, D. J. 1981. "A Method for Estimating the Probability of Adverse Drug Reactions." Clin. Pharmacol. Ther. 30: 239-45.

[9] Kim, K. A., Chung, J., Jung, D. H., and Park, J. Y. 2004. "Identification of Cytochrome P450 Isoforms Involved in the Metabolism of Loperamide in Human Liver Microsomes.” Eur. J. Clin. Pharmacol. 60: 575-81.

[10] Schinkel, A.H., Wagenaar, E., Mol, C.A., and van Deemter, L. 1996. "P-Glycoprotein in the Blood-Brain Barrier of Mice Influences the Brain Penetration and Pharmacological Activity of Many Drugs.” J. Clin. Invest. 97: 2517-24.

[11] Montesinos, R. N., Moulari, B., Gromand, J., Beduneau, A., Lamprecht, A., and Pellequer, Y. 2014. "Coadministration of P-Glycoprotein Modulators on Loperamide Pharmacokinetics and Brain Distribution." Drug Metab. Dispos. 42: 700-6.

[12] Sadeque, A. J., Wandel, C., He, H., Shah, S., and Wood, A. J. 2000. "Increased Drug Delivery to the Brain by P-Glycoprotein Inhibition." Clin. Pharmacol. Ther. 68: 231-7.

[13] Eggleston, W., Nacca, N., and Marraffa, J. M. 2015. "Loperamide Toxicokinetics: Serum Concentrations in the Overdose Setting." Clin. Toxicol. 53: 495-6.

[14] Antzelevitch, C. 2005. "Role of Transmural Dispersion of Repolarization in the Genesis of Drug-Induced Torsades de Pointes." Heart Rhythm 2: S9-15.

[15] Al-Khatib, S. M., LaPointe, N. M., Kramer, J. M., and Califf, R. M. 2003. "What Clinicians Should Know About the QT Interval." JAMA 289: 2120-7. 